\title{
Chemotherapy-Related Leukemia
}

National Cancer Institute

\section{Source}

National Cancer Institute. Chemotherapy-Related Leukemia. NCI Thesaurus. Code C80691.

A leukemia arising as a result of the mutagenic effect of chemotherapy agents that are used for the treatment of a malignant tumor. 